\title{
Contagem dupla
}

\author{
Edney Freitas Gregório (1) Luiz Augustavo Almeida Feitoza (1)
}

\section{Resumo}

Este pequeno artigo tem por objetivo apresentar o método Contagem Dupla como recurso a ser utilizado em provas e demonstrações de algumas afirmações matemáticas. São construídos vários argumentos por meio de problemas práticos, e suas soluções fornecem-nos demonstrações para resultados matemáticos, por vezes não triviais, fazendo uso de contagem dupla. A relevância do estudo reside no fato de uma ideia extremamente simples - ancorada a soluções interessantes para alguns problemas - proporcionarem-nos demonstrações para afirmações não triviais.

Palavras-chave: Contagem Dupla; Progressões Aritméticas; Progressões Geométricas; Soma de Potências de Inteiros Positivos.

\begin{abstract}
This short article aims to present the Double Counting method as a resource to use in proof and to use some mathematical statements. Various arguments are constructed for practical problems in the middle, and their solutions are applied to mathematical results, sometimes not trivial, using double counting. The relevance of the study lies in the fact that it is an extremely simple idea, anchored in interesting solutions to some problems, which are provided for non-trivial statements.
\end{abstract}

Keywords: Double Counting; Arithmetic Progressions; Geometric Progressions; Sum of Powers of Positive Integers.

\section{Introdução}

O desenvolvimento de habilidades relativas a argumentação para defesa de nossas ideias, é uma característica humana que passa a ocorrer já na infância, quando, por exemplo, naturalmente busca-se convencer familiares, como pais e irmãos, sobre algo que se deseja conseguir.

Durante toda a vida desenvolvemos, até mesmo involuntariamente, mecanismos que nos auxiliam na atividade de apresentar nossas ideias, das mais variadas e em diferentes cenários, em busca de persuadir o máximo possível de pessoas.

No meio científico, desenvolver habilidades sobre argumentação para convencer a comunidade científica de que suas afirmações são verdadeiras, é fundamental. E, por essa razão, muitos estudos são desenvolvidos continuamente sobre métodos que possibilitem segurança quanto à comprovação de determinada afirmação nova. A grande obra O Discurso do Método, de René Descartes, é um exemplo dos esforços humanos de sistematizar métodos de racionalidade aceitável para comprovação de premissas postas. 
Nesse aspecto, a Matemática constitui-se como uma ciência fértil em relação a métodos de comprovações de suas conjecturas. É natural em Matemática formularmos afirmações e buscarmos, por meios que possibilitem, mostrar, provar ou demostrar que elas fazem sentido e que são justificáveis.

No presente texto apresentamos um método extremamente interessante para demonstrações de algumas afirmações matemáticas, denominado método de Contagem Dupla. Iniciamos discorrendo sobre o resultado matemático que alicerça o método, muito embora a ideia que justifica sua validade seja extremamente simples.

Em seguida, enunciamos alguns problemas práticos seguidos respectivamente de suas soluções, as quais serão vislumbradas como prova para afirmações matemáticas bem mais gerais.

Vale observar que, para uma boa compreensão dos resultados aqui apresentados, é importante o leitor ter relativa familiaridade com conjuntos, operações sobre os mesmos, funções e análise combinatória.

\section{Contagem Dupla}

Neste tópico apresentamos o resultado basilar da argumentação que garante a eficácia do método de Contagem Dupla. Inicialmente vejamos as seguintes notações.

Para $n \in \mathbb{N}$, denotamos por $\mathrm{I}_{\mathrm{n}}$ o conjunto $\{1,2,3, \cdots, \mathrm{n}\}=\mathrm{I}_{\mathrm{n}}$ dos números naturais de 1 a $\mathrm{n}$.

Um conjunto $\mathrm{A} \neq \varnothing$ é finito se existe uma bijeção $\mathrm{f}: \mathrm{I}_{\mathrm{n}} \rightarrow \mathrm{A}$, para algum $\mathrm{n} \in \mathbb{N}$. Nesse caso denotamos $a_{j}=f(j)$, e escrevemos $A=\left\{a_{1}, a_{2}, \cdots, a_{n}\right\}$. Dizemos que $n$ é o número de elementos do conjunto A e denotamos por $|\mathrm{A}|=\mathrm{n}$ ou $\# \mathrm{~A}=\mathrm{n}$. Depreendemos então que uma bijeção $\mathrm{f}: \mathrm{I}_{\mathrm{n}} \rightarrow \mathrm{A}$ é uma contagem dos elementos de $\mathrm{A}$.

Iremos mostrar que se exitem bijeções $\mathrm{f}: \mathrm{I}_{\mathrm{n}} \rightarrow \mathrm{A}, \mathrm{g}: \mathrm{I}_{\mathrm{m}} \rightarrow \mathrm{A}, \mathrm{n}, \mathrm{m} \in \mathbb{N}$, então $\mathrm{n}=\mathrm{m}$. Isso significa que se realizarmos a contagem dos elementos de um conjunto finito não vazio, de dois modos diferentes, encontraremos o mesmo resultado. É claro que intuitivamente tal afirmação é óbvia, no entanto, necessitamos sistematizar essa ideia matematicamente, com o rigor adequado.

Seguindo as notações do parágrafo anterior, observe que ao considerarmos a função $h=g^{-1} \circ f$ : $I_{n} \rightarrow I_{m}$, basta mostrar que se existe uma bijeção $h: I_{n} \rightarrow I_{m}$, então $n=m$, que faremos por meio da proposição a seguir.

Teorema 1. Se existe uma bijeção $\psi: \mathrm{I}_{\mathrm{n}} \rightarrow \mathrm{I}_{\mathrm{m}}, \mathrm{n}, \mathrm{m} \in \mathbb{N}$, então $\mathrm{n}=\mathrm{m}$.

Demonstração: Utilizaremos indução sobre n. Para $n=1$, como $\psi$ é bijeção temos que $I_{m}$ possui um único elemento, ou seja: $m=1$. Suponhamos pois que $n>1$ e consideremos uma bijeção $\phi: \mathrm{I}_{\mathrm{n}+1} \rightarrow \mathrm{I}_{\mathrm{m}+1}$, tal que $\phi(\mathrm{n}+1)=\mathrm{m}+1$. A restrição de $\phi$ a $\mathrm{I}_{\mathrm{n}}$ dá-nos uma bijeção $\psi: \mathrm{I}_{\mathrm{n}} \rightarrow \mathrm{I}_{\mathrm{m}}$. Logo, por hipótese tempos que $n=m$, e consequentemente $n+1=m+1$, o que conclui a prova pelo Princípio da Indução Finita.

A técnica de Contagem Dupla consiste na simples ideia de que se calcularmos a quantidade de "configurações" (elementos) de um conjunto finito e não vazio de duas maneiras distintas sem cometer erros, então obteremos o mesmo resultado. Vejamos a técnica sendo utilizada no resultado a seguir.

Proposição 1. Se $\mathrm{n}$ e $\mathrm{k}$ são inteiros positivos tais que $0 \leq \mathrm{k} \leq \mathrm{n}$, então, todo conjunto que possui $\mathrm{n}$ elementos possui exatamente $\left(\begin{array}{l}\mathrm{n} \\ \mathrm{k}\end{array}\right)$ subconjuntos com $\mathrm{k}$ elementos. 
Prova: Seja $\mathrm{C}_{\mathrm{k}}^{\mathrm{n}}$ a quantidade de subconjuntos de k elementos do conjunto A. Como $\mathrm{C}_{0}^{\mathrm{n}}=\left(\begin{array}{l}\mathrm{n} \\ 0\end{array}\right)=1$, consideremos $1 \leq \mathrm{k} \leq \mathrm{n}$.

Fixemos um conjunto A com n elementos e consideremos o problema de contar a quantidade de sequências de $\mathrm{k}$ termos formada a partir dos elementos desse conjunto.

Primeira contagem: pela fórmula dos arranjos sem repetição, a resposta é

$$
\mathrm{n} \cdot(\mathrm{n}-1) \cdots(\mathrm{n}-\mathrm{k}+1) \text {. }
$$

Segunda Contagem: permutando os elementos de cada subconjunto de $\mathrm{k}$ elementos do conjunto A, obtemos k! sequências de k termos. Note que as sequências assim formadas são todas distintas, e são todas que podemos obter a partir de $\mathrm{k}$ elementos de um subconjunto de A. Portanto, há exatamente $\mathrm{k} ! \cdot \mathrm{C}_{\mathrm{k}}^{\mathrm{n}}$ sequências de $\mathrm{k}$ elementos.

Mas desde que $\mathrm{n} \cdot(\mathrm{n}-1) \cdots(\mathrm{n}-\mathrm{k}+1)$ e $\mathrm{k} ! \cdot \mathrm{C}_{\mathrm{k}}^{\mathrm{n}}$ são respostas para um mesmo problema, concluímos que

$$
\mathrm{k} ! \cdot \mathrm{C}_{\mathrm{k}}^{\mathrm{n}}=\mathrm{n} \cdot(\mathrm{n}-1) \cdots(\mathrm{n}-\mathrm{k}+1) .
$$

Daí,

$$
\mathrm{C}_{\mathrm{k}}^{\mathrm{n}}=\frac{\mathrm{n} \cdot(\mathrm{n}-1) \cdots(\mathrm{n}-\mathrm{k}+1)}{\mathrm{k} !}=\frac{\mathrm{n} \cdot(\mathrm{n}-1) \cdots(\mathrm{n}-\mathrm{k}+1) \cdot(\mathrm{n}-\mathrm{k}) !}{\mathrm{k} ! \cdot(\mathrm{n}-\mathrm{k}) !}=\left(\begin{array}{l}
\mathrm{n} \\
\mathrm{k}
\end{array}\right)
$$

\section{Progressões Aritméticas e Progressões Geométricas}

Nesta seção, veremos que por meio da solução de um problema específico, demonstramos um resultado relativo a determinados tipos de progressões aritméticas, e em seguida, de modo análogo, demonstramos um resultado relativo a progressões geométricas.

Consideremos o seguinte problema:

Problema 1. Em uma festa com n pessoas, sorteia-se uma pessoa para escolher um entre a prêmios, ou duas pessoas, para escolherem um entre $\mathbf{r}$ prêmios. Deseja-se descobrir de quantas formas esse sorteio pode ser realizado.

Soluções: Faremos duas contagens da mesma situação.

Primeira contagem: o primeiro sorteio é realizado escolhendo-se uma pessoa entre n e depois 1 prêmio entre a possíveis. O segundo sorteio é realizado escolhendo-se 2 de n pessoas, e depois 1 de $\boldsymbol{r}$ prêmios. Os dois eventos são independentes, logo, o total de modos de escolha é

$$
\left(\begin{array}{l}
n \\
1
\end{array}\right) \cdot a+\left(\begin{array}{l}
n \\
2
\end{array}\right) \cdot r
$$

Segunda contagem: denotemos as pessoas por $\mathrm{P}_{1}, \mathrm{P}_{2}, \cdots, \mathrm{P}_{\mathrm{n}}$. Para cada pessoa sorteada, no primeiro sorteio, há a escolhas de prêmios. Assim, há a escolhas para cada $\mathrm{i} \in\{1,2, \cdots, \mathrm{n}\}$. No segundo sorteio, é formado um par $\left(\mathrm{P}_{\mathrm{i}}, \mathrm{P}_{\mathrm{j}}\right)$, onde a ordem não importa, logo podemos supor que $\mathrm{i} \leq \mathrm{j}$. Como uma pessoa não pode formar par consigo, podemos escrever $\mathrm{i}<\mathrm{j}$. Os possíveis pares são $\left(\mathrm{P}_{1}, \mathrm{P}_{\mathrm{i}}\right), \cdots,\left(\mathrm{P}_{\mathrm{i}-1}, \mathrm{P}_{\mathrm{i}}\right)$, ou seja, temos $\mathrm{i}-1$ pares. Cada par de pessoas escolhe um dentre $\boldsymbol{r}$ prêmios, logo, há para esse sorteio $\mathrm{r} \cdot(\mathrm{i}-1)$ possibilidades. Considerando-se os dois sorteios, para cada $\mathrm{i}$ temos $\mathrm{a}_{\mathrm{i}}=\mathrm{a}+\mathrm{r} \cdot(\mathrm{i}-1)$ possibilidades. O total de possibilidades é, portanto, $\sum_{\mathrm{i}=1}^{\mathrm{n}} \mathrm{a}_{\mathrm{i}}$, ou seja, a soma dos n primeiros termos da progressão aritmética com termo inicial a e razão $\boldsymbol{r}$. 
Notemos que a solução do Problema 1 fornece uma demonstração para o Teorema 2 a seguir.

Teorema 2. A soma

$$
\sum_{i=1}^{n} a_{i}
$$

dos $\mathrm{n}$ primeiros termos de uma progressão aritmética de razão $\mathrm{r} \in \mathbb{Z}, \mathrm{r}>0$, e primeiro termo $\mathrm{a} \in \mathbb{Z}, \mathrm{a}>0$, é igual $a$

$$
\mathrm{a} \cdot\left(\begin{array}{l}
\mathrm{n} \\
1
\end{array}\right)+\mathrm{r} \cdot\left(\begin{array}{l}
\mathrm{n} \\
2
\end{array}\right) .
$$

Problema 2. Temos uma quantidade suficientemente grande de lâmpadas de q-1 tipos. Queremos colocar essas lâmpadas em a ruas, onde cada rua tem $\boldsymbol{n}$ postes, a princípio sem lâmpadas. Desejamos fazer isso de modo que em cada rua pelo menos um dos postes tenha uma lâmpada. De quantas formas essa atividade pode ser realizada?

Soluções: Assim como no Problema 1, faremos a contagem, de dois modos distintos, da quantidade de maneiras de realizar esta atividade.

Primeira contagem: fixemos uma rua qualquer. Para cada poste dessa rua, temos $(q-1)+1$ possibilidades, pois podemos colocar em um poste uma das q-1 lâmpadas, ou podemos deixar o poste sem lâmpada. Como são $n$ postes, temos $\mathrm{q}^{\mathrm{n}}$ possibilidades ao todo, das quais devemos excluir a configuração em que nenhum poste tem lâmpada. Logo, temos $q^{n}-1$ possibilidades para cada rua. Como são a ruas, ao todo são a $\cdot\left(q^{n}-1\right)$ configurações diferentes para todas as ruas.

Segunda contagem: consideremos uma rua qualquer, fixemos um poste i e escolhamos uma lâmpada do tipo k para esse poste. Nos postes que vêm antes do poste i, coloquemos uma lâmpada qualquer ou não coloquemos lâmpada alguma (q possibilidades para cada um dos i-1 postes). No poste i coloquemos a lâmpada do tipo k (uma possibilidade). E nos postes que vêm depois do poste i, não coloquemos lâmpada alguma (uma possibilidade para cada um dos n - i postes). Assim, temos

$$
\underbrace{\mathrm{q} \cdot \mathrm{q} \cdots \mathrm{q} \cdot \mathrm{q}}_{\mathrm{i}-1} \cdot \underbrace{1}_{\text {tipo } \mathrm{k}} \cdot \underbrace{1 \cdot 1 \cdots 1 \cdot 1}_{\mathrm{n}-\mathrm{i}}=\mathrm{q}^{\mathrm{i}-1}
$$

modos de colocar lâmpadas em uma rua onde no poste i tem a lâmpada do tipo k. Como temos a ruas, o número de possibilidades para todas as ruas onde no poste i tem a lâmpada do tipo k é

$$
\mathrm{a} \cdot \mathrm{q}^{\mathrm{i}-1}=\mathrm{a}_{\mathrm{i}}, \quad 1 \leq \mathrm{i} \leq \mathrm{n},
$$

ou seja,

$$
\sum_{i=1}^{n} a_{i} \text { modos. }
$$

Uma vez que a lâmpada fixada no poste i pode ser de qualquer um dos q-1 tipos, o número total de possibilidades para todas as ruas é

$$
(q-1) \cdot \sum_{i=1}^{n} a_{i}
$$

Realizamos, portanto, duas contagens diferentes para o mesmo conjunto de possibilidades, e com isso obtemos a igualdade

$$
a \cdot\left(q^{n}-1\right)=(q-1) \cdot \sum_{i=1}^{n} a_{i},
$$

que é o resultado do teorema a seguir. 
Teorema 3. A soma

$$
\sum_{i=1}^{n} a_{i}
$$

dos $\mathrm{n}$ primeiros termos de uma progressão geométrica de razão $\mathrm{q} \in \mathbb{Z}, \mathrm{q}>1$, e primeiro termo $\mathrm{a} \in \mathbb{Z}, \mathrm{a}>0$, é igual $a$

$$
\mathrm{a} \cdot \frac{\mathrm{q}^{\mathrm{n}}-1}{\mathrm{q}-1}
$$

\section{Soma de Potências}

Nesta seção apresentamos a demonstração de um resultado interessante sobre somas de potências de números inteiros não negativos, utilizando contagens duplas. Vamos iniciar provando, abaixo, soma de uma quantidade finita de cubos. Faremos isso, por meio da solução do problema abaixo.

Problema 3. Deseja-se calcular a quantidade de elementos do conjunto

$$
\mathbb{S}=\left\{\left(\mathrm{a}_{1}, \mathrm{a}_{2}, \mathrm{a}_{3}, \mathrm{a}_{4}\right) \in \mathbb{Z}^{4}: 0 \leq \mathrm{a}_{1}, \mathrm{a}_{2}, \mathrm{a}_{3}<\mathrm{a}_{4} \leq \mathrm{n}\right\}
$$

Soluções: Solucionaremos o problema de dois modos distintos.

Primeira contagem: seja k o maior elemento de uma quádrupla ordenada de $\mathbb{S}$. Devemos escolher três termos não necessariamente distintos entre $0,1,2, \cdots, \mathrm{k}-1$ para formar a quádrupla, e isso pode ser feito de $\mathrm{k} \cdot \mathrm{k} \cdot \mathrm{k} \cdot 1=\mathrm{k}^{3}$ modos, para cada $\mathrm{k} \in\{1,2, \cdots, \mathrm{n}\}$, ou seja, $\sum_{\mathrm{k}=1}^{\mathrm{n}} \mathrm{k}^{3}$, modos. Portanto, $\mathbb{S}$ possui $\sum_{\mathrm{k}=1}^{\mathrm{n}} \mathrm{k}^{3}$ elementos.

Segunda contagem: contemos todas as quádruplas ordenadas de $\mathbb{S}$ que tem exatamente i elementos distintos $(2 \leq \mathrm{i} \leq 4)$.

$\mathbf{i}=2$ : temos $\mathrm{C}_{2}^{\mathrm{n}+1}$ modos de escolher os termos $\mathrm{a}_{3}$ e $\mathrm{a}_{4}$. O penúltimo termo $\left(\mathrm{a}_{3}\right)$ aparece repetido três vezes na quádrupla, o que nos dá

$$
\mathrm{C}_{2}^{\mathrm{n}+1} \cdot \frac{3 !}{3 !}=\left(\begin{array}{c}
\mathrm{n}+1 \\
2
\end{array}\right) \cdot 1=\left(\begin{array}{c}
\mathrm{n}+1 \\
2
\end{array}\right) \operatorname{modos} .
$$

Observe que podemos escrever $\frac{3 !}{3 !}=\sum_{\substack{\mathrm{k}_{1}=3 \\ \mathrm{k}_{1} \in \mathbb{N}}} \frac{3 !}{\mathrm{k}_{1} !}$

$\mathbf{i}=3$ : temos $\mathrm{C}_{3}^{\mathrm{n}+1}$ maneiras de escolher os 3 elementos distintos que irão aparecer na quádrupla. O maior deles será o $a_{4}$, e os outros dois termos devem ser usados para preencher os 3 lugares restantes, assim um deles deverá aparecer duas vezes. Isso nos dá

$$
\mathrm{C}_{3}^{\mathrm{n}+1} \cdot\left(\frac{3 !}{1 ! \cdot 2 !}+\frac{3 !}{2 ! \cdot 1 !}\right)=\left(\begin{array}{c}
\mathrm{n}+1 \\
3
\end{array}\right) \cdot \sum_{\substack{\mathrm{k}_{1}+\mathrm{k}_{2}=3 \\
\mathrm{k}_{1}, \mathrm{k}_{2} \in \mathbb{N}}} \frac{3 !}{\mathrm{k}_{1} ! \cdot \mathrm{k}_{2} !}=6 \cdot\left(\begin{array}{c}
\mathrm{n}+1 \\
3
\end{array}\right) \text { modos. }
$$

O termo $\left(\frac{3 !}{1 ! \cdot 2 !}+\frac{3 !}{2 ! \cdot 1 !}\right)$ é resultado da permutação com repetição. 
$\mathbf{i}=4$ : temos $\mathrm{C}_{4}^{\mathrm{n}+1}$ modos de escolher os quatro termos distintos e obviamente cada termo aparecerá uma única vez. Isso nos dá

$$
\mathrm{C}_{4}^{\mathrm{n}+1} \cdot \frac{3 !}{1 ! \cdot 1 ! \cdot 1 !}=\left(\begin{array}{c}
\mathrm{n}+1 \\
4
\end{array}\right) \cdot \sum_{\substack{\mathrm{k}_{1}+\mathrm{k}_{2}+\mathrm{k}_{3}=3 \\
\mathrm{k}_{1}, \mathrm{k}_{2}, \mathrm{k}_{3} \in \mathbb{N}}} \frac{3 !}{\mathrm{k}_{1} ! \cdot \mathrm{k}_{2} ! \cdot \mathrm{k}_{3} !}=6 \cdot\left(\begin{array}{c}
\mathrm{n}+1 \\
4
\end{array}\right) \text { modos. }
$$

Assim como no caso anterior, o termo $\frac{3 !}{1 ! \cdot 1 ! \cdot 1 !}$ é resultado da permutação com repetição.

Somando as quantidades calculadas em cada caso acima, obtemos um total de elementos de $\mathbb{S}$ ou seja,

$$
\left(\begin{array}{c}
n+1 \\
2
\end{array}\right)+6 \cdot\left[\left(\begin{array}{c}
n+1 \\
3
\end{array}\right)+\left(\begin{array}{c}
n+1 \\
4
\end{array}\right)\right]
$$

As duas contagens distintas utilizadas para calcularmos a quantidade de elementos de $\mathbb{S}$ no problema anterior fornece-nos uma demonstração do resultado abaixo.

Teorema 4. Para $\mathrm{n} \in \mathbb{Z}, \mathrm{n} \geq 0$, temos

$$
\sum_{\mathrm{k}=1}^{\mathrm{n}} \mathrm{k}^{3}=\left(\begin{array}{c}
\mathrm{n}+1 \\
2
\end{array}\right)+6 \cdot\left[\left(\begin{array}{c}
\mathrm{n}+1 \\
3
\end{array}\right)+\left(\begin{array}{c}
\mathrm{n}+1 \\
4
\end{array}\right)\right]
$$

Vejamos agora uma generalização da ideia do Teorema 4, por meio de duas soluções distintas do problema a seguir.

Problema 4. Deseja-se calcular a quantidade de elementos do conjunto $\mathbb{P}$ definido da seguinte forma

$$
\mathbb{P}=\left\{\left(\mathrm{a}_{1}, \cdots, \mathrm{a}_{\mathrm{m}}, \mathrm{a}_{\mathrm{m}+1}\right) \in \mathbb{Z}^{\mathrm{m}+1}: 0 \leq \mathrm{a}_{1}, \cdots, \mathrm{a}_{\mathrm{m}}<\mathrm{a}_{\mathrm{m}+1} \leq \mathrm{n}\right\} .
$$

Soluções: Mais uma vez faremos duas soluções para o mesmo problema.

Primeira contagem: seja k o maior elemento de uma $(\mathrm{m}+1)$-upla ordenada de $\mathbb{P}$. Devemos escolher $\mathrm{m}$ termos não necessariamente distintos entre $0,1,2, \cdots, \mathrm{k}-1$ para formar a $(\mathrm{m}+1)-$ upla. Isso pode ser feito de $\underbrace{\mathrm{k} \cdot \mathrm{k} \cdot \mathrm{k} \cdots \mathrm{k}}_{\mathrm{m}} \cdot 1=\mathrm{k}^{\mathrm{m}}$ modos, para cada $\mathrm{k} \in\{1,2, \cdots, \mathrm{n}\}$, ou seja, $\sum_{\mathrm{k}=1}^{\mathrm{n}} \mathrm{k}^{\mathrm{m}}$, modos. Portanto, $\mathbb{P}$ possui $\sum_{\mathrm{k}=1}^{\mathrm{n}} \mathrm{k}^{\mathrm{m}}$ elementos.

Segunda contagem: contemos todas as $(m+1)$-uplas ordenadas de $\mathbb{P}$ que tem exatamente i elementos distintos $(2 \leq \mathrm{i} \leq \mathrm{m}+1)$.

Primeiro devemos escolher quem serão os i termos distintos, o que pode ser feito de

$$
\left(\begin{array}{c}
n+1 \\
\mathrm{i}
\end{array}\right) \operatorname{modos}
$$

Sabendo quem são os termos distintos, escolhemos o maior deles para ficar na última entrada da $(\mathrm{m}+1)$-upla, e os $\mathrm{i}-1$ termos restantes devem ser usados para preencher as $\mathrm{m}$ entradas restantes. 
Podemos repetir qualquer um dos $\mathrm{i}-1$ termos. Consideremos $\mathrm{k}_{1}, \mathrm{k}_{2}, \cdots, \mathrm{k}_{\mathrm{i}-1}$ suas multiplicidades. Logo, usando permutação com repetição temos

$$
\sum_{\mathrm{k}_{1}+\mathrm{k}_{2}+\cdots+\mathrm{k}_{\mathrm{i}-1}=\mathrm{m}} \frac{\mathrm{m} !}{\mathrm{k}_{1} ! \cdot \mathrm{k}_{2} ! \cdots \mathrm{k}_{\mathrm{i}-1} !} \text { modos, }
$$

com $\mathrm{k}_{1}, \mathrm{k}_{2}, \cdots, \mathrm{k}_{\mathrm{i}-1} \geq 1$, pois cada um dos $\mathrm{i}-1$ termos aparecem pelo menos uma vez e $\mathrm{k}_{1}+\mathrm{k}_{2}+$ $\cdots+\mathrm{k}_{\mathrm{i}-1}=\mathrm{m}$ já que devemos preencher as $\mathrm{m}$ entradas restantes.

Portanto, a quantidade de elementos do conjunto $\mathbb{P}$ é

$$
\sum_{\mathrm{i}=2}^{\mathrm{m}+1}\left(\begin{array}{c}
\mathrm{n}+1 \\
\mathrm{i}
\end{array}\right) \cdot \sum_{\substack{\mathrm{k}_{1}+\mathrm{k}_{2}+\cdots+\mathrm{k}_{\mathrm{i}-1}=\mathrm{m} \\
\mathrm{k}_{1}, \mathrm{k}_{2}, \cdots, \mathrm{k}_{\mathrm{i}-1} \in \mathbb{N}}} \frac{\mathrm{m} !}{\mathrm{k}_{1} ! \cdot \mathrm{k}_{2} ! \cdots \mathrm{k}_{\mathrm{i}-1} !}
$$

As soluções acima do Problema 4, obtidas por meio das duas contagens, dão-nos uma demonstração do teorema abaixo.

Teorema 5. Para $\mathrm{n}, \mathrm{m} \in \mathbb{Z}, \mathrm{n} \geq 1, \mathrm{~m} \geq 1$ vale a seguinte identidade

$$
\sum_{\mathrm{k}=1}^{\mathrm{n}} \mathrm{k}^{\mathrm{m}}=\sum_{\mathrm{i}=2}^{\mathrm{m}+1}\left(\begin{array}{c}
\mathrm{n}+1 \\
\mathrm{i}
\end{array}\right) \cdot \sum_{\mathrm{k}_{1}+\mathrm{k}_{2}+\cdots+\mathrm{k}_{\mathrm{i}-1}=\mathrm{m}} \frac{\mathrm{m} !}{\mathrm{k}_{1} ! \cdot \mathrm{k}_{2} ! \cdots \mathrm{k}_{\mathrm{i}-1} !},
$$

com $\mathrm{k}_{1}, \mathrm{k}_{2}, \cdots, \mathrm{k}_{\mathrm{i}-1} \in \mathbb{N}$.

A soma de potências de números inteiros positivos já foi objeto de estudo de vários matemáticos ao longo da história. No texto intitulado Sums of Powers of Positive Integers, de autoria da professora Janet Beery (Universidade de Redlands, Califórnia), é apontada uma lista dos matemáticos que se debruçaram sobre o problema, além de serem apresentadas as ideias centrais de seus estudos. No mesmo artigo são propostos e solucionados alguns problemas envolvendo somas de potências. Veja mais em [2].

Não encontramos em nossas pesquisas nenhum documento que disponha uma demonstração do Teorema 5 como essa que apresentamos neste trabalho, por meio da Contagem Dupla realizada para solucionar o Problema 4.

\section{Considerações finais}

Como vimos, foram apresentados alguns problemas práticos, cujas soluções, realizadas com duas contagens distintas, permitiram-nos vislumbrar identidades interessantes, sendo as duas contagens o suficiente para a demonstração desses resultados.

Ressaltamos que um conjunto muito variado de identidades matemáticas podem ser demonstradas por meio de contagens duplas.

Este texto é parte de um estudo maior acerca de demonstrações de resultados matemáticos utilizando essa técnica. Acreditamos ser uma abordagem interessante e esperamos que este trabalho possa suscitar outros estudos nesse sentido. 


\section{Referências}

[1] Andreescu, T. e Feng, Z. A Path to Combinatorics for Undergraduates: Counting Strategies. Birkhauser, 2003.

[2] Beery, J. Sums of Powers of Positive Integers. University of Redlands, 2010. Disponível em: https://www.maa.org/press/periodicals/convergence/sums-of-powers-of-positive-integers. Acesso em: 08 de abril de 2020.

[3] Chuan-Chong, C. e Khee-Meng, K. Principles and Techniques in Combinatorics. World Scientific, 1992.

[4] Morgado, A. C; Pitombeira, J. B; Pinto Carvalho, P. C. e Fernandez, P. Análise Combinatória e Probabilidade. Rio de Janeiro-RJ: Sociedade Brasileira de Matemática (SBM), 2016.

[5] Muniz Neto, A. C. Tópicos de Matemática Elementar - Volume 4 Combinatória. Rio de Janeiro/RJ: Sociedade Brasileira de Matemática (SBM), 2016.

Edney Freitas Gregório Universidade Estadual do Ceará (Uece) Campus Itaperi <edney.gregorio@uece.br>

Luiz Augustavo Almeida Feitoza Instituto Federal de Educação, Ciência e Tecnologia do Amazonas Campus Avançado Iranduba (Ifam/CIR) <luiz.feitoza@ifam.edu.br>

Recebido: $13 / 04 / 2020$

Publicado: 21/09/2020 